\title{
S Research Square \\ Paclitaxel Combined with Ticagrelor Inhibits B16F10 and Lewis Lung Carcinoma Cell Metastasis
}

\section{Xingjun Meng}

Suzhou Municipal Hospital

\section{Zhihui Cao}

Suzhou Municipal Hospital

\section{Renfeng Liu}

Suzhou Municipal Hospital

Kai Zheng

Suzhou Municipal Hospital

Shuai Ding

Suzhou Municipal Hospital

Yuefan Gu

Suzhou Municipal Hospital

Yonghua Chen

Suzhou Municipal Hospital

Jun Lv

Suzhou Municipal Hospital

Ping Li

Suzhou Municipal Hospital

Li Zhou

Suzhou Municipal Hospital

\section{Wenbo Wang}

Suzhou Municipal Hospital

\section{Shiliang Ji}

Suzhou Municipal Hospital

Hui He

Suzhou Municipal Hospital

Hui Yang ( $\nabla$ yanghui_sci@163.com )

Suzhou Municipal Hospital

\section{Research Article}

Keywords: paclitaxel, ticagrelor, tumor metastasis, B16F10, Lewis Lung carcinoma 
Posted Date: October 27th, 2021

DOl: https://doi.org/10.21203/rs.3.rs-960139/v1

License: (c) (i) This work is licensed under a Creative Commons Attribution 4.0 International License. Read Full License 


\section{Abstract}

It is widely accepted that tumor metastasis is the dominant factor leading to cancer-related death. Tumor metastasis is mediated by cell invasion, blood circulation and lymphatic circulation. Paclitaxel, as a common anti-tumor drug and a mitotic inhibitor, promotes microtubule assembly and inhibits microtubule depolymerization. In addition, ticagrelor, an anti-platelet drug, is used to treat acute coronary syndrome. An increasing numbers of studies have reported that platelets can facilitate tumor metastasis. Therefore, inhibiting the effects of platelets can serve as a novel therapeutic strategy for cancer. To explore the effect of anti-tumor and anti-platelet drugs on tumor progression, the murine melanoma cell line, B16F10, and Lewis Lung carcinoma (LLC) cells were treated with paclitaxel and/or ticagrelor. Interestingly, the results demonstrated that paclitaxel and ticagrelor could not only suppress the proliferation, migration and invasion of B16F10 and LLC cells, but they could also prevent tumor metastasis to the lungs. Furthermore, the inhibitory effect of paclitaxel and ticagrelor was more apparent when both drugs were used in combination. Collectively, the current study demonstrated that the combination of paclitaxel and ticagrelor could be considered as a potential anti-tumor therapy approach.

\section{Introduction}

As the leading cause of cancer-related deaths, tumor metastasis is considered to be the key target of tumor therapy ${ }^{1}$. In the 19 th century, a study showed that the interaction between platelets and tumor cells was closely associated with thrombosis and malignant tumors ${ }^{2}$. Recently, it has been suggested that the number of platelets is positively associated with tumor metastasis ${ }^{3}$. Another study demonstrated that depleting platelets or blocking their function could significantly prevent tumor metastasis, thus supporting the key role of platelets in tumor metastasis ${ }^{4}$. However, the mechanisms underlying the effects of platelets on tumor metastasis remain to be uncovered.

Paclitaxel, a natural anti-cancer drug, has been extensively used in the clinical treatment of breast, ovarian, head and neck, and lung cancer ${ }^{5-7}$. However, paclitaxel is not considered as the ideal treatment approach for melanoma, since only $16 \%$ of patients with melanoma have some palliative benefit from paclitaxel therapy ${ }^{8}$. Melanoma is a type of skin melanocyte-derived cancer, characterized by high degree of malignancy, rapid metastasis and poor prognosis. Although the incidence of melanoma is only $3 \%$ among all malignant tumors, $80 \%$ of skin-related cancer types may lead to death due to their high degree of malignancy ${ }^{9}$. Surgical resection, chemotherapy and immunotherapy are common strategies for treating melanoma; however, these treatment approaches are accompanied by several side effects ${ }^{10,11}$. Since melanoma is not sensitive to paclitaxel and the number of functional platelets plays a significant role on tumor metastasis, the present study aimed to investigate whether the combined therapy with paclitaxel and the anti-platelet drug, ticagrelor, could be an effective treatment strategy for melanoma. Ticagrelor was approved by the Food and Drug Administration (FDA) in 2011 for the treatment of acute coronary syndrome via targeting the platelet $\mathrm{P} 2 \mathrm{Y} 12$ receptor ${ }^{12}$. A recent study reported that ticagrelor could inhibit human and murine breast cancer cell metastasis via blocking the platelet-tumor cell 
interaction ${ }^{13}$. Another study revealed that ticagrelor could prevent cell metastasis and improve survival in different mouse tumor models ${ }^{14}$.

Emerging evidence has suggested the platelets are associated with tumor metastasis ${ }^{15}$. Therefore, inhibition of platelet function could be considered as a novel strategy for attenuating tumor metastasis. Our previous study demonstrated that ticagrelor prevented tumor metastasis via inhibiting B16F10 and Lewis lung carcinoma (LLC) cell proliferation and promoting platelet apoptosis ${ }^{16}$. Therefore, the present study aimed to further explore the effects of ticagrelor and paclitaxel on tumor progression.

For more than half a century, B16F10 and LLC cells have been widely used in oncology research due to their high tumorigenic rate and rapid metastasis ${ }^{17-19}$. Herein, B16F10 and LLC cells were treated with the combination of paclitaxel and ticagrelor to investigate cell viability, motility and invasion ability in vitro. In addition, in vivo experiments were carried out to verify the efficacy of the combination of both drugs against tumor metastasis. The results showed that the combination of paclitaxel and ticagrelor not only inhibited the proliferation, migration and invasion of B16F10 and LLC cells, but also prevented tumor cell metastasis to the lungs. Overall, the current study revealed that the combination of paclitaxel and ticagrelor exhibited a synergistic anti-tumor effect on B16F10 and LLC cells in vitro and in vivo. These findings indicated that the combination of anti-tumor and anti-platelet drugs could be considered as a novel approach for inhibiting B16F10 and LLC cell metastasis to the lungs.

\section{Materials And Methods}

Cell lines and mice. The murine melanoma cell line, B16F10, and Lewis Lung carcinoma (LLC) cells were purchased from the Chinese Academy of Sciences Cell Bank. C57BL/6 mice (males; age, 6-8 weeks, number. 40) were obtained from SPF (Beijing) Biotechnology Co., Ltd. All animal experiments were approved by the Ethics Committee of the Suzhou Institute of Biomedical Engineering and Technology, Chinese Academy of Science.

Chemicals and regents. Paclitaxel (cat. no. S1150) and ticagrelor (cat. no. S4079) were purchased from Selleck Chemicals, and prepared as 5 or $20 \mathrm{mM}$ stock solution, and stored at $-80^{\circ} \mathrm{C}$. FBS (cat. no. $10270-$ 106), penicillin/streptomycin solution (cat. no. 2029632) and high glucose DMEM (cat. no. 10313021) were obtained from Gibco; Thermo Fisher Scientific, Inc. The Annexin V-FITC kit (cat. no. LHK601-100) was obtained from Jiamay Biotech Co., Ltd., while the Cell Counting Kit-8 (CCK-8; cat. no. GB707) from Dojindo Molecular Technologies, Inc. The Matrigel ${ }^{T \mathrm{M}}$ Basement Membrane Matrix (cat. no. 354234; BD Biosciences) was obtained from Corning, Inc., and the antibodies against Bad (ca. no. sc-8044) and Bax (cat. no. sc-493) from Santa Cruz Biotechnology, Inc. Finally, the anti-caspase-3 (cat. no. A0214), antiGAPDH (cat. no. AC035), HRP Goat Anti-mouse IgG (cat. no. AS003) and HRP Goat Anti-Rabbit IgG (cat. no. AS014) antibodies were purchased from ABclonal Biotech Co., Ltd.

Cell viability assay. Cells were grown in culture flasks, harvested in trypsin/EDTA solution, and resuspended in DMEM supplemented with $2 \%$ FBS. Subsequently, a total of 5,000 cells were seeded into 
96-well plates. Following incubation for $6 \mathrm{~h}$, cells were treated with paclitaxel $(5 \mathrm{nM})$, ticagrelor $(20 \mu \mathrm{M})$ or paclitaxel $(5 \mathrm{nM})$ combined with ticagrelor $(20 \mu \mathrm{M})$. Each experiment was repeated for four times per group independently. The cell viability assay was performed according to the manufacturer's instructions (CCK-8; cat. no. GB707; Dojindo Molecular Technologies, Inc). Briefly, $10 \mu \mathrm{l}$ CCK-8 reagent was added into each well and cells were cultured for $48 \mathrm{~h}$. Following incubation for $2 \mathrm{~h}$ at $37^{\circ} \mathrm{C}$, the optical density (OD) at $450 \mathrm{~nm}$ was measured in each well.

Platelet isolation. C57/BL6 wild-type mice (ag, 6-8 weeks) were anesthetized by intraperitoneal injection of $2 \%$ pentobarbital sodium $(130 \mathrm{mg} / \mathrm{kg}$,) and fixed by pin. Following anesthesia for $5 \mathrm{~min}$, fresh blood was collected (1 ml/mouse) from the inferior vena cava using a $1 / 7$ volume of acid-citrate-dextrose $(2.5 \%$ trisodium citrate, $2.0 \%$ d-glucose, $1.5 \%$ citric acid) as anticoagulant. When needed, mice were sacrificed by cervical dislocation. Subsequently, the blood was centrifuged at $200 \mathrm{xg}$ for $10 \mathrm{~min}$ and the supernatant was transferred into new tubes and centrifuged at $800 \mathrm{xg}$ for $3 \mathrm{~min}$. The precipitate was then washed with CGS buffer $(0.9 \% \mathrm{NaCl}, 0.03 \mathrm{M}$ d-glucose, $0.01 \mathrm{M}$ trisodium citrate, $\mathrm{pH} 7.0)$ and resuspended in modified Tyrode's buffer (MTB; $12 \mathrm{mM} \mathrm{NaHCO}_{3}, 0.9 \% \mathrm{NaCl}, 5.6 \mathrm{mM}$ d-glucose, $2.6 \mathrm{mM}$ $\mathrm{KCl}, 2.4 \mathrm{mM}$ HEPES, $1 \mathrm{mM} \mathrm{CaCl}_{2}, 1 \mathrm{mM} \mathrm{MgCl}_{2}$, and $0.1 \% \mathrm{BSA}, \mathrm{pH}$ 7.4). The platelets were counted using the Sysmex XP-100 hematology analyzer (Sysmex Co.) and incubated at room temperature for $1 \mathrm{~h}$.

Co-culture of B16F10 or LLC cells with platelets. Cells were harvested from culture flasks after trypsinization (with EDTA) and resuspended in DMEM containing $2 \%$ FBS. Subsequently, $2 \times 10^{5}$ cells/well were seeded into 6 -well culture plates. Following incubation for $4 \mathrm{~h}$, the prepared platelets $\left(2 \times 10^{6} /\right.$ well) were added into each well and cultured for an additional $48 \mathrm{~h}$. Subsequently, cells/platelets were treated with paclitaxel $(5 \mathrm{nM})$, ticagrelor $20 \mu \mathrm{M}$ or paclitaxel $(5 \mathrm{nM})+$ ticagrelor $(20 \mu \mathrm{M})$.

Colony formation assay. Cells were harvested from culture flasks by trypsinization and resuspended in DMEM supplemented with $10 \%$ FBS. Subsequently, $500 \mu \mathrm{l}$ (300 cells) of the cell suspension were added into 12-well plates. Following incubation for $4 \mathrm{~h}$, cells were treated with paclitaxel $(5 \mathrm{nM})$, ticagrelor $(20$ $\mu \mathrm{M})$ or paclitaxel $(5 \mathrm{nM})+$ ticagrelor $(20 \mu \mathrm{M})$. Cells in the control group were treated with DMSO. The medium was replaced every 4 days. After culture for 12 days, the medium was removed and cells were washed with PBS for three times. Finally, for Giemsa staining, cells were fixed with methanol.

Wound healing assay. For wound healing assay, $2 \times 10^{5}$ cells/well were seeded into 6-well plates. The next day, wounds were created by scratching using 1-ml pipette tip. Following washing twice with PBS, the wells were supplemented with $2 \mathrm{ml}$ DMEM (with $2 \% \mathrm{FBS}$ ). To evaluate cell migration, images were captured at three fields from each wound at 0 and $48 \mathrm{~h}$ under a microscope.

Transwell assay. For Transwell assays, the membrane of the 24-well Transwell plates was pre-coated with $100 \mu \mathrm{l}$ Matrigel for $2 \mathrm{~h}$ at $37^{\circ} \mathrm{C}$. Cells in culture flasks were harvested using $1 \mathrm{ml}$ trypsin/EDTA solution and resuspended in DMEM containing $1 \%$ FBS to stop digestion. Subsequently, $100 \mu \mathrm{l}\left(1 \times 10^{5}\right.$ cells) of the cell suspension was added into the upper chamber of the 24-well Transwell plate. The lower chamber was supplemented with $700 \mu \mathrm{LMEM}$ and incubated for $48 \mathrm{~h}$ at $37^{\circ} \mathrm{C}$. Following incubation, the 
membrane was removed from the 24-well plate, and the invasive cells were fixed with methanol and stained with $0.2 \%$ crystal violet solution after washing with PBS.

Phosphatidylserine (PS) externalization assay. Isolated platelets $\left(1 \times 10^{7}\right)$ were treated with paclitaxel (5 $\mathrm{nM})$, ticagrelor $(20 \mu \mathrm{M})$ or paclitaxel $(5 \mathrm{nM})+$ ticagrelor $(20 \mu \mathrm{M})$. Following treatment, the cells were mixed with Annexin V-binding buffer (1X) and Annexin V-FITC (50:50:1) for $1 \mathrm{~h}$. The cell samples were shaken gently and analyzed by flow cytometry after $15 \mathrm{~min}$.

Western blot analysis. Platelets were lysed by RIPA buffer (cat. no. WB3100), the isolated protein samples were subjected to SDS-PAGE and transferred to PVDF membranes. The membranes were then incubated with primary antibodies against Bad, Bax, caspase-3 and GAPDH (reference gene). Images of the protein bands were captured using the Tanon-5200 Multi system (Tanon Science and Technology Co., Ltd.). Data were semi-quantified using the Image $J$ software V1.8.0 (National Institues oof Health).

Tumor mouse models. In the current study two tumor models were established in C57BL/6 mice. Briefly, $3 \times 10^{5} \mathrm{~B} 16 \mathrm{~F} 10$ or $5 \times 10^{5} \mathrm{LLC}$ cells in $50 \mu \mathrm{l}$ PBS were injected into the tail vein of 5 mice/group. Mice were treated with paclitaxel $(10 \mathrm{mg} / \mathrm{kg})$ or ticagrelor $(10 \mathrm{mg} / \mathrm{kg})$ every 2 days by tail vein injection for 14 days for the B16F10 model or 20 days for the LLC model. On day 15 (B16F10 model) or 21 (LLC model), mice were sacrificed by cervical dislocation. Subsequently, the lungs were collected and the number of metastatic lung nodules, with a size of 0.2-1.5 mm, was recorded.

Statistical analysis. All statistical analyses were carried out utilizing Student's t-test or one-way ANOVA with the SPSS 19.0 software (IBM Corp.). All data are expressed as the mean \pm standard deviation (SD; $\mathrm{n} \geq 3$ ). All $\mathrm{P}$-values were two-sided and $\mathrm{P}<0.05$ was considered to indicate a statistically significant difference.

\section{Results}

Combination of paclitaxel with ticagrelor inhibits the proliferation of B16F10 and LLC cells. To investigate the effect of paclitaxel and ticagrelor on the proliferation of B16F10 and LLC cells, drug toxicity was evaluated using a CCK-8 assay. Firstly, the $\mathrm{IC}_{50}$ values of paclitaxel and ticagrelor were determined (Fig. S1), and the appropriate concentration was selected for the subsequent experiments. The results showed that cell viability was decreased by 15 and $31 \%$ in B16F10 cells treated with $20 \mu \mathrm{M}$ ticagrelor or $5 \mathrm{nM}$ paclitaxel, respectively (Fig. 1A). In addition, the B16F10 cell proliferation ability was also evaluated using colony formation assays. Therefore, the number of colonies was reduced by 33 and $49 \%$ in cells treated with ticagrelor or paclitaxel, respectively (Fig. 1B). Furthermore, when cells were co-treated with ticagrelor and paclitaxel, the cell viability was decreased by $51 \%$, and the colony formation rate by $67 \%$ (Fig. 1B and C). These findings suggested that both paclitaxel and ticagrelor could inhibit cell proliferation, and that ticagrelor combined with paclitaxel exerted a synergistic effect on B16F10 cell proliferation. Additionally, the results in the LLC cell line were consistent with those observed in B16F10 cells. 
Combination of paclitaxel with ticagrelor attenuates the migratory and invasive abilities of B16F10 and $L L C$ cells. Previous studies have demonstrated that platelets can facilitate tumor progression. Herein, to explore the effect of ticagrelor and paclitaxel on cell mobility and invasiveness, wound healing and Transwell assays were implemented. The results showed that platelets promoted cell migration when cocultured with B16F10 and LLC cells; however, this effect was reversed following treatment with ticagrelor and/or paclitaxel (Fig. 2A and B). Similarly, the Transwell assays showed that the number of cells invading the cell matrix membrane was increased after platelet stimulation, while ticagrelor and paclitaxel restored this effect (Fig. 2C and D). The abovementioned results indicated that platelets could enhance the migration and invasion ability of B16F10 and LLC cells, which was diminished following treatment with ticagrelor and paclitaxel. Besides, when paclitaxel was combined with ticagrelor, the inhibitory effect on cell migration and invasion was more remarkable.

Combination of paclitaxel with ticagrelor promotes platelet apoptosis. To investigate the effects of paclitaxel and ticagrelor on platelets, mouse platelets were isolated from anticoagulated whole blood and then treated with ticagrelor, paclitaxel, or their combination. PS externalization assay was used to assess cell apoptosis. The platelet apoptosis rate (PS\%) was 4.4 and $5.4 \%$ in platelets treated with $20 \mu \mathrm{M}$ ticagrelor or $5 \mathrm{nM}$ paclitaxel, respectively (Fig. $3 \mathrm{~A}$ and B). However, the apoptosis rate was increased to $18.4 \%$ when platelets were treated with the combination of paclitaxel and ticagrelor (Fig. $3 A$ and B). In addition, the expression levels of pro-apoptotic proteins were determined by western blot analysis.

Therefore, when platelets were treated with either paclitaxel or ticagrelor, the expression levels of Bax, Bad and caspase- 3 were notably upregulated. Treatment with combination of paclitaxel and ticagrelor further increased the expression levels of the pro-apoptotic proteins (Fig. 3C-F). The results demonstrated that paclitaxel as well as ticagrelor could promote platelet apoptosis, which was further increased when the two drugs were used.

Combination of paclitaxel with ticagrelor inhibits lung metastasis in mice. The effects of paclitaxel and ticagrelor were further verified in vivo. B16F10 or LLC cells were injected into C57BL/ 6 mice via the tail vein. The following day, paclitaxel and ticagrelor were administrated. Lungs were obtained at 15 (B16F10 model) or 21 days (LLC model) post administration to count the number of metastatic lung nodules. Since the LLC model was not sufficient enough to observe metastatic lung nodules, H\&E staining was utilized. The normal lungs had many alveoli, which were reduced when neoplastic tissue was present. The results demonstrated that the number of metastatic lung nodules was significantly reduced in the paclitaxel- and ticagrelor-treated groups compared with the vehicle group (Fig. 4A-C). Furthermore, fewer cells were metastasized to the lungs when the combination of both drugs was applied. Collectively, paclitaxel and ticagrelor could attenuate B16F10 and LLC cell metastasis to the lungs in mice.

\section{Discussion}

As early as 140 years ago, studies have shown that platelets are non-nucleated cell elements, originating from bone marrow megakaryocytes, and cleared in the reticuloendothelial system ${ }^{20}$. Thrombosis and hemostasis are the fundamental functions of platelets. In recent years, it has been reported that platelets 
are involved in tumor metastasis. Platelets are not only considered to be the "umbrella" of tumor cells, but can also promote tumor cell dissemination ${ }^{21}$. Following the detachment from the primary tumor, tumor cells migrate to the distal end through lymphatic or blood circulation ${ }^{22}$. When tumor cells escape from the primary tumor and penetrate the blood vessels, platelets are then responsible for their survival. Therefore, platelets aggregate to the surface of tumor cells to form a physical barrier, thus protecting tumor cells against the blood shear stress-mediated damage ${ }^{23}$. At the same time, platelet markers and major platelet histocompatibility complex $\otimes$ are transferred to the surface of tumor cells, thus providing protection against the $T$ lymphocyte- and natural killer-mediated immunosurveillance ${ }^{21}$. In addition, studies have shown that tumor cell platelet aggregates secrete large amounts of growth factors, cytokines and other factors that promote the activation of endothelial cells ${ }^{24}$. In turn, activated endothelial cells produce E-selectin, P-selectin and vascular cell adhesion protein 1, which bind and block their ligands on tumor cells to adhere to blood vessels. Moreover, activated platelets secrete chemokines, such as $\mathrm{C}-\mathrm{C}$ motif chemokine ligand 5 , to further recruit monocytes to the surface of tumor cells, thus promoting metastasis ${ }^{25}$. A recent study revealed that platelet-guided early metastasis started within $2 \mathrm{~h}$ after tumor cell blocking, thus providing a special tumor microenvironment, which is crucial for the subsequent metastatic process ${ }^{26}$. Additionally, another study indicated that platelet-tumor cell aggregates and platelet-secreted TGF- $\beta$ could act synergistically to activate the TGF- $\beta /$ Smad $\beta$ and NF-KB signaling pathways, which in turn promoted tumor cell invasion and metastasis ${ }^{4}$.

In total, $\sim 90 \%$ of cancer-related mortality is attributed to metastases, and it has been suggested that several factors are involved in this process, including genetic factors, stem cells, growth factors, platelets and cell-cell interactions ${ }^{1}$. Tumor metastasis mainly occurs via direct tumor cell invasion, and blood and lymphatic circulation, while the lymphatic vasculature has been regarded as a bridge for tumor dissemination 27,28 . A study suggested that platelets could play a vital role in blood-borne metastasis ${ }^{20}$. In addition, a previous study from our laboratory demonstrated that B16F10 cells could metastasize to the lungs through the blood circulation. Furthermore, neither subcutaneous nor intraperitoneal injection were applied within two weeks after lung metastasis ${ }^{29}$. The aforementioned studies indicated that platelets could serve as an ideal target for inhibiting tumor metastasis through the blood circulation. In 2016, the United States Preventive Services Task Force recommended the initiation of low-dose aspirin for the primary prevention of colorectal cancer in a particular patient population ${ }^{30}$. This recommendation has set a precedent for the application of anti-platelet drugs in the prevention and treatment of tumors. Currently, several studies have focused on the application of anti-platelet drugs, such as aspirin, clopidogrel and ticagrelor in the treatment of different types of cancer ${ }^{31-33}$.

Overall, platelets play a positive role in tumor growth and metastasis. Although platelet clearance can inhibit tumor growth and metastasis, this approach has no clinical application value since it may lead to life-threatening bleeding ${ }^{34}$. Inhibiting the function of platelets or blocking the interaction between platelets and tumor cells could prevent tumor progression. Herein, the effects of the anti-platelet drug, ticagrelor, combined with the anti-tumor drug, paclitaxel, were investigated in B16F10 and LLC cells. The 
results suggested that the combination of both drugs could significantly attenuate tumor metastasis, thus providing a novel approach for anti-tumor therapy. However, the clinical application of both drugs needs to be further investigated.

\section{Abbreviation}

LLC: Lewis Lung carcinoma

FDA: Food and Drug Administration

SPF: Specific Pathogen Free

CCK-8: Cell Counting Kit-8

OD: Optical density

MTB: Modified Tyrode's Buffer

PS: Phosphatidylserine

PVDF: Polyvinylidene Fluoride

SIBET: Suzhou Institute of Biomedical Engineering and Technology

\section{Declarations}

\section{Acknowledgements}

The animal experiments were performed at the Suzhou Institute of Biomedical Engineering and Technology (SIBET), Chinese Academy of Science.

\section{Funding}

This study was supported by the Suzhou Science \& Technology Plan (grant nos. KJXW2019035 and SYSD2019167); Research project of Jiangsu Pharmaceutical Association Tianqing hospital pharmaceutical Foundation (Q202022).

\section{Availability of data and materials}

All data generated or analyzed during this study are included in this published article. If you are interested in the data, please contact the following person.

Hui Yang

Affiliation: The Suzhou Hospital Affiliated of Nanjing Medical University 
Mailing address: Suzhou Municipal Hospital, 16 West Baita Street, Suzhou, 215002, Jiangsu Province, China

E-mail: yanghui_sci@163.com

Xingjun Meng

Affiliation: School of Life Sciences, Nanjing University

Mailing address:163 Xianlin Avenue, Xixia District, Nan Jing, Jiangsu Province, China

E-mail: mxj89647385@163.com

\section{Author's contributions}

$\mathrm{XM}, \mathrm{ZC}$ and RL made substantial contributions to conception and design of the current study, interpretation of data and performed the statistical analyses. $Y G, Y C, J L, P L, L Z$, and WW performed the animal experiments. $\mathrm{KZ}$ and SD edited the manuscript. $\mathrm{HH}$ and $\mathrm{HY}$ supported and supervised the whole project. All authors read and approved the final manuscript.

\section{Ethics approval and consent to participate}

Animal experiments were authorized by the Ethics Committee of the Suzhou Institute of Biomedical Engineering and Technology (SIBET), Chinese Academy of Science.

\section{Patient consent for publication}

Not applicable.

\section{Competing interests}

The authors declare that they have no competing interests.

\section{References}

1. Qiu W, Su GH. Challenges and advances in mouse modeling for human pancreatic tumorigenesis and metastasis. Cancer Metastasis Rev. Jun 2013;32(1-2):83-107. doi:10.1007/s10555-012-9408-2

2. Jurasz P, Alonso-Escolano D, Radomski MW. Platelet--cancer interactions: mechanisms and pharmacology of tumour cell-induced platelet aggregation. Br J Pharmacol. Dec 2004;143(7):81926. doi:10.1038/sj.bjp.0706013

3. Erdemir F, Kilciler M, Bedir S, Ozgok Y, Coban H, Erten K. Clinical significance of platelet count in patients with renal cell carcinoma. Urol Int. 2007;79(2):111-116. doi:10.1159/000106322

4. Labelle M, Begum S, Hynes RO. Direct Signaling between Platelets and Cancer Cells Induces an Epithelial-Mesenchymal-Like Transition and Promotes Metastasis. Cancer Cell. Nov 15 
2011;20(5):576-590. doi:10.1016/j.ccr.2011.09.009

5. Woo HL, Swenerton KD, Hoskins PJ. Taxol is active in platinum-resistant endometrial adenocarcinoma. Am J Clin Oncol-Canc. Jun 1996;19(3):290-291. doi:Doi 10.1097/00000421199606000-00016

6. Stathopoulos GP, Rigatos S, Papakostas P, Fountzilas G. Effectiveness of paclitaxel and carboplatin combination in heavily pretreated patients with head and neck cancers. Eur J Cancer. Oct 1997;33(11):1780-1783. doi:Doi 10.1016/S0959-8049(97)00200-1

7. Brower V. Afatinib and chemotherapy in non-small-cell lung cancer. Lancet Oncol. Feb 2016;17(2):E47-E47. doi:10.1016/S1470-2045(15)00609-9

8. Wiernik PH, Einzig Al. Taxol in malignant melanoma. J Natl Cancer Inst Monogr. 1993;(15):185-7.

9. Dzwierzynski WW. Managing malignant melanoma. Plast Reconstr Surg. Sep 2013;132(3):446e-60e. doi:10.1097/PRS.0b013e31829ad411

10. Kaiser S, Vassell R, Pinckney RG, Holmes TE, James TA. Clinical impact of biopsy method on the quality of surgical management in melanoma. J Surg Oncol. Jun 2014;109(8):775-9. doi:10.1002/jso.23580

11. Mukherjee N, Schwan JV, Fujita M, Norris DA, Shellman YG. Alternative Treatments For Melanoma: Targeting BCL-2 Family Members to De-Bulk and Kill Cancer Stem Cells. J Invest Dermatol. Sep 2015;135(9):2155-2161. doi:10.1038/jid.2015.145

12. Mitrugno A, McCarty OJT. Ticagrelor breaks up the tumor-platelet party. Blood. Sep 7 2017;130(10):1177-1178. doi:10.1182/blood-2017-07-795898

13. Gareau AJ, Brien C, Gebremeskel S, Liwski RS, Johnston B, Bezuhly M. Ticagrelor inhibits platelettumor cell interactions and metastasis in human and murine breast cancer. Clin Exp Metastasis. Feb 2018;35(1-2):25-35. doi:10.1007/s10585-018-9874-1

14. Gebremeskel S, LeVatte T, Liwski RS, Johnston B, Bezuhly M. The reversible P2Y12 inhibitor ticagrelor inhibits metastasis and improves survival in mouse models of cancer. International Journal of Cancer. Jan 1 2015;136(1):234-240. doi:10.1002/ijc.28947

15. Elaskalani O, Berndt MC, Falasca M, Metharom P. Targeting Platelets for the Treatment of Cancer. Cancers (Basel). Jul 22 2017;9(7)doi:10.3390/cancers9070094

16. Meng $X$, Liu W, Yang H, et al. Ticagrelor prevents tumor metastasis via inhibiting cell proliferation and promoting platelet apoptosis. Anticancer Drugs. Nov 2020;31(10):1012-1017.

doi:10.1097/CAD.0000000000000925

17. Gerber SA, Sorensen EW, Sedlacek AL, et al. Local expression of interleukin-2 by B16 melanoma cells results in decreased tumour growth and long-term tumour dormancy. Immunology. Mar 2013;138(3):280-92. doi:10.1111/imm.12037

18. Bertram JS, Janik P. Establishment of a cloned line of Lewis Lung Carcinoma cells adapted to cell culture. Cancer Lett. Nov 1980;11(1):63-73. doi:10.1016/0304-3835(80)90130-5 
19. Nakamura K, Yoshikawa N, Yamaguchi Y, Kagota S, Shinozuka K, Kunitomo M. Characterization of mouse melanoma cell lines by their mortal malignancy using an experimental metastatic model. Life Sciences. Jan 4 2002;70(7):791-798. doi:Doi 10.1016/S0024-3205(01)01454-0

20. Franco AT, Corken A, Ware J. Platelets at the interface of thrombosis, inflammation, and cancer. Blood. Jul 30 2015;126(5):582-588. doi:10.1182/blood-2014-08-531582

21. Kopp HG, Placke T, Salih HR. Platelet-derived transforming growth factor-beta down-regulates NKG2D thereby inhibiting natural killer cell antitumor reactivity. Cancer Res. Oct 1 2009;69(19):777583. doi:10.1158/0008-5472.CAN-09-2123

22. Labelle M, Hynes RO. The initial hours of metastasis: the importance of cooperative host-tumor cell interactions during hematogenous dissemination. Cancer Discov. Dec 2012;2(12):1091-9. doi:10.1158/2159-8290.CD-12-0329

23. Egan K, Cooke N, Kenny D. Living in shear: platelets protect cancer cells from shear induced damage. Clin Exp Metastasis. Aug 2014;31(6):697-704. doi:10.1007/s10585-014-9660-7

24. Koupenova M, Clancy L, Corkrey HA, Freedman JE. Circulating Platelets as Mediators of Immunity, Inflammation, and Thrombosis. Circ Res. Jan 19 2018;122(2):337-351. doi:10.1161/Circresaha.117.310795

25. Qian BZ, Li JF, Zhang H, et al. CCL2 recruits inflammatory monocytes to facilitate breast-tumour metastasis. Nature. Jul 14 2011;475(7355):222-U129. doi:10.1038/nature10138

26. Labelle M, Hynes RO. The Initial Hours of Metastasis: The Importance of Cooperative Host-Tumor Cell Interactions during Hematogenous Dissemination. Cancer Discovery. Dec 2012;2(12):10911099. doi:10.1158/2159-8290.Cd-12-0329

27. Sleeman JP. The lymph node as a bridgehead in the metastatic dissemination of tumors. Recent Results Canc. 2000;157:55-81.

28. Sleeman JP, Thiele W. Tumor metastasis and the lymphatic vasculature. Int J Cancer. Dec 15 2009;125(12):2747-56. doi:10.1002/ijc.24702

29. Meng X, Xiaodong LI, Liu J, et al. Establishment of a C57BL/ $6 \mathrm{~J}$ mouse model of metastatic melanoma in the lung. Acta Laboratorium Animalis Scientia Sinica. 2018;

30. Bibbins-Domingo K, Force UPST. Aspirin Use for the Primary Prevention of Cardiovascular Disease and Colorectal Cancer: US Preventive Services Task Force Recommendation Statement. Ann Intern Med. Jun 21 2016;164(12):836-U103. doi:10.7326/M16-0577

31. Xu XR, Yousef GM, Ni H. Cancer and platelet crosstalk: opportunities and challenges for aspirin and other antiplatelet agents. Blood. Apr 19 2018;131(16):1777-1789. doi:10.1182/blood-2017-05743187

32. Yu LX, Yan L, Yang W, et al. Platelets promote tumour metastasis via interaction between TLR4 and tumour cell-released high-mobility group box1 protein. Nat Commun. Oct 2014;5doi:ARTN $52561038 /$ ncomms6256

33. Zhang YL, Wei JY, Liu SL, et al. Inhibition of platelet function using liposomal nanoparticles blocks tumor metastasis. Theranostics. 2017;7(5):1062-1071. doi:10.7150/thno.17908 


\section{Figures}
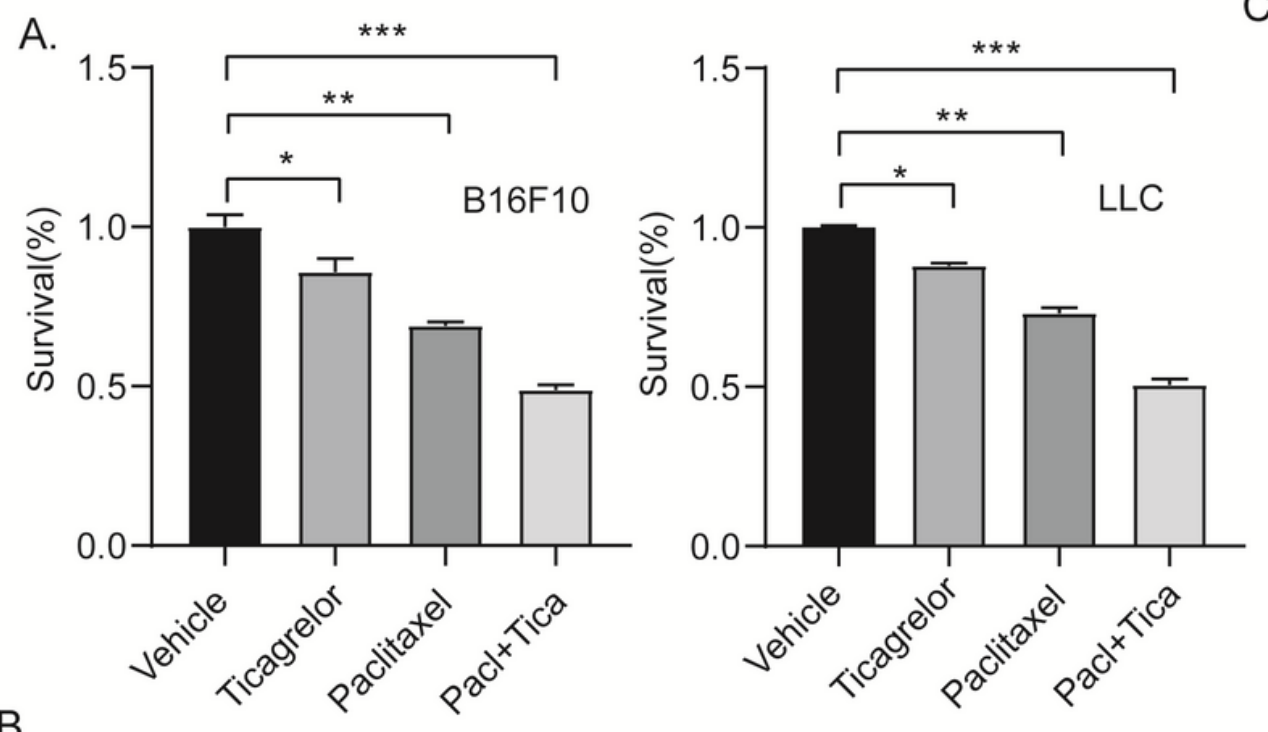

C.

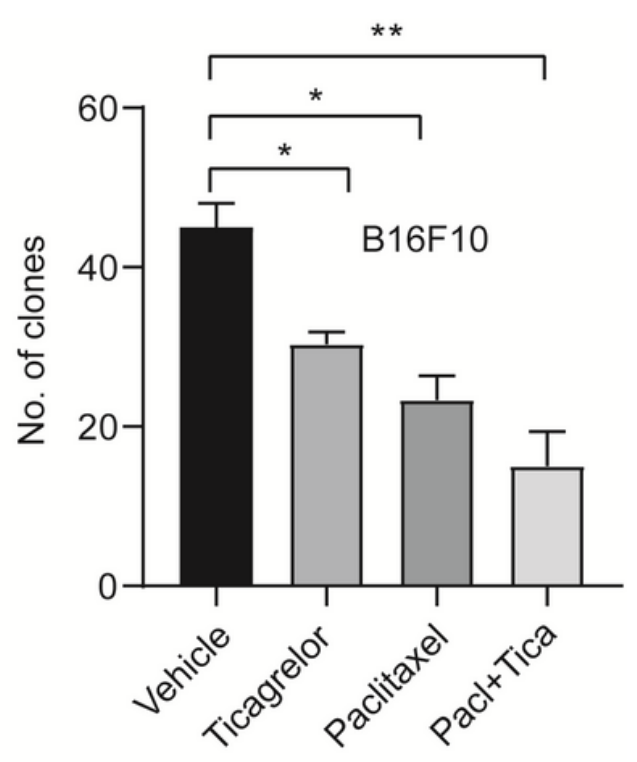

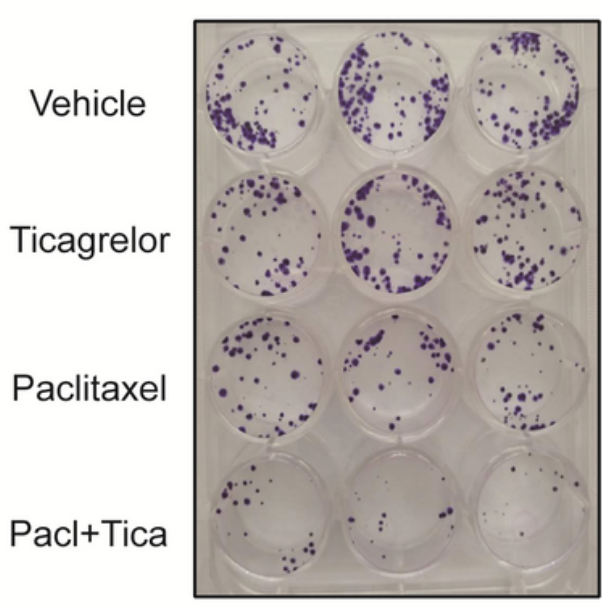

B16F10

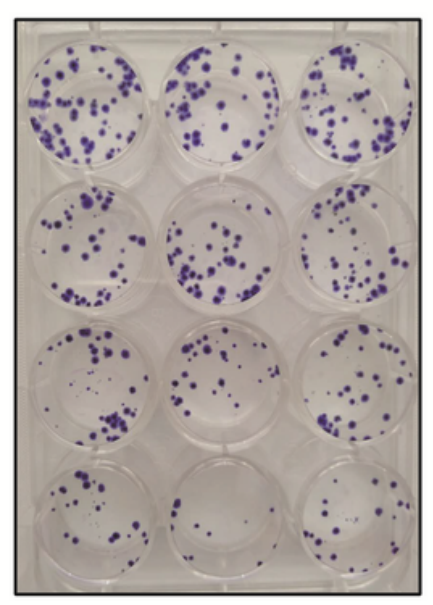

LLC
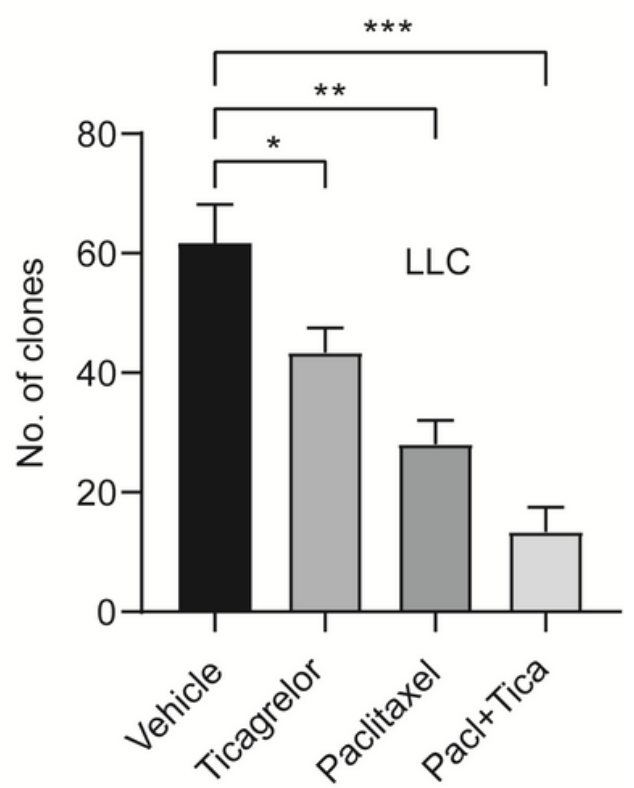

\section{Figure 1}

Combination of paclitaxel with ticagrelor inhibits the proliferation of B16F10 and LLC cells. (A) The cell viability of B16F10 and LLC cells treated with $5 \mathrm{nM}$ paclitaxel or $20 \mu \mathrm{M}$ ticagrelor or paclitaxel $(5 \mathrm{nM})+$ ticagrelor $(20 \mu \mathrm{M})$ for $48 \mathrm{~h}$ was evaluated by CCK-8 assay. ${ }^{*} \mathrm{P}<0.05$, ${ }^{* \star} \mathrm{P}<0.01$ and ${ }^{* \star *} \mathrm{P}<0.001$ vs. control group. (B, C) B16F10 and LLC cells were treated with $5 \mathrm{nM}$ paclitaxel or $20 \mu \mathrm{M}$ ticagrelor or paclitaxel (5 $\mathrm{nM})+$ ticagrelor $(20 \mu \mathrm{M})$ or DMSO (control) and cultured for 12 days. Cells were then fixed with methanol and stained with Giemsa. ${ }^{*} P<0.05,{ }^{*} \mathrm{P}<0.01$ and ${ }^{*} * \mathrm{P}<0.001$ vs. control group. LLC, Lewis lung carcinoma; CCK-8, Cell Counting Kit 8. 

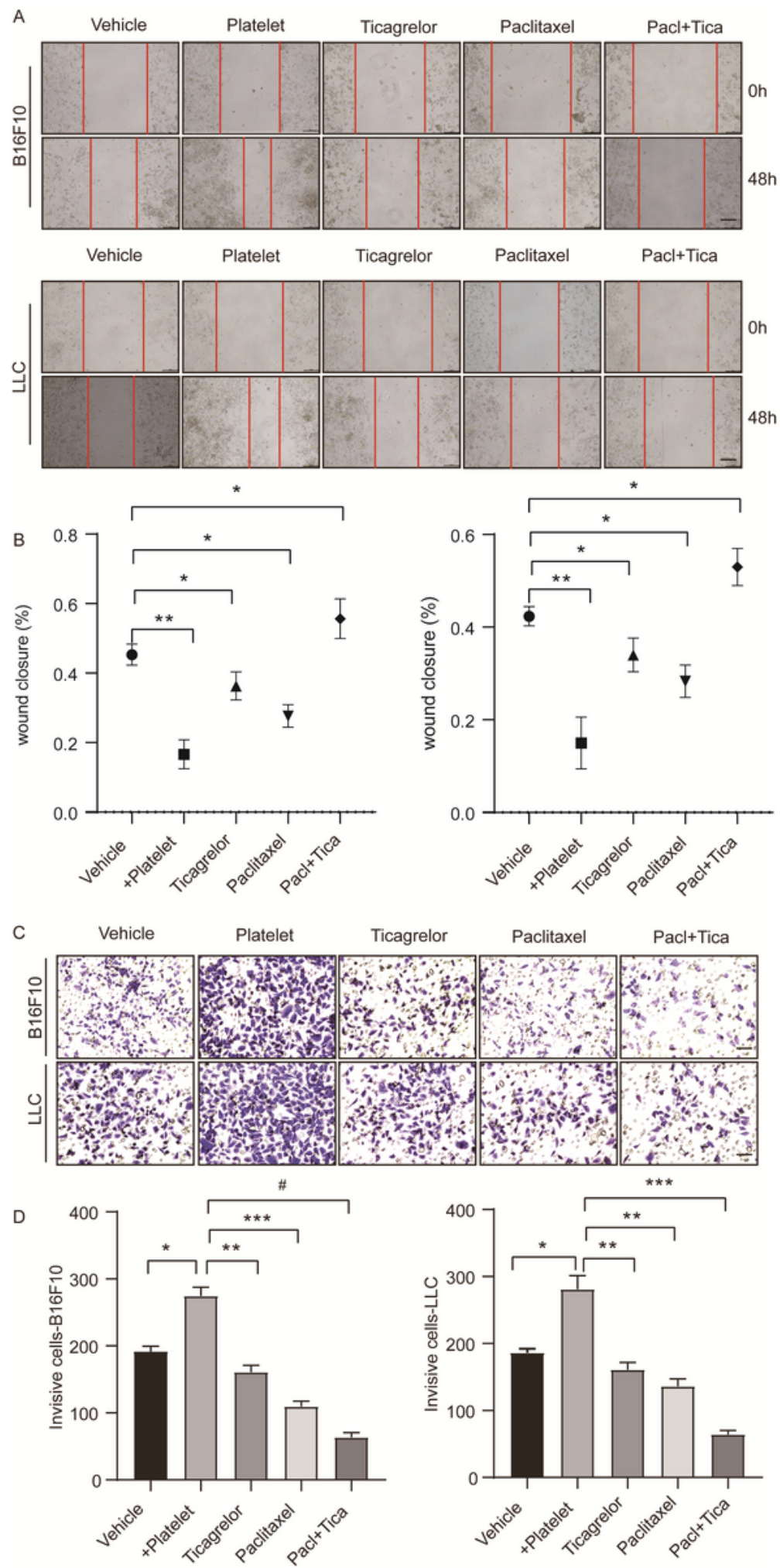

Figure 2

Combination of paclitaxel with ticagrelor attenuates the migratory and invasive abilities of B16F10 and LLC cells. (A, B) B16F10 or LLC cell monolayers (density, 2x105) were scratched, and cells were then cocultured with platelets (density, 2x106); platelets and $5 \mathrm{nM}$ paclitaxel; platelets and $20 \mu \mathrm{M}$ ticagrelor; or platelets and $5 \mathrm{nM}$ paclitaxel $+20 \mu \mathrm{M}$ ticagrelor. Following incubation for $48 \mathrm{~h}$, cells in the different treatment groups were observed under a microscope. Data were analyzed using the ImageJ software. 
Data are expressed as the mean \pm standard deviation (SD; $n=3) .{ }^{*}<0.05$ and ${ }^{*} P<0.01$ vs. the platelet group. (C, D) Transwell invasion assay results in the abovementioned treatment groups are shown (scale bar, $100 \mu \mathrm{m})$. The cells in $\mathrm{C}$ were stained with crystal violet and measured using the Image J software. Data are expressed as the mean \pm standard deviation (SD; $n=3$ ). ${ }^{*}<<0.05, * \star P<0.01$ and ${ }^{*} * * P<0.001$ vs. the platelet group; $\# \mathrm{P}<0.0001$. LLC, Lewis lung carcinoma.
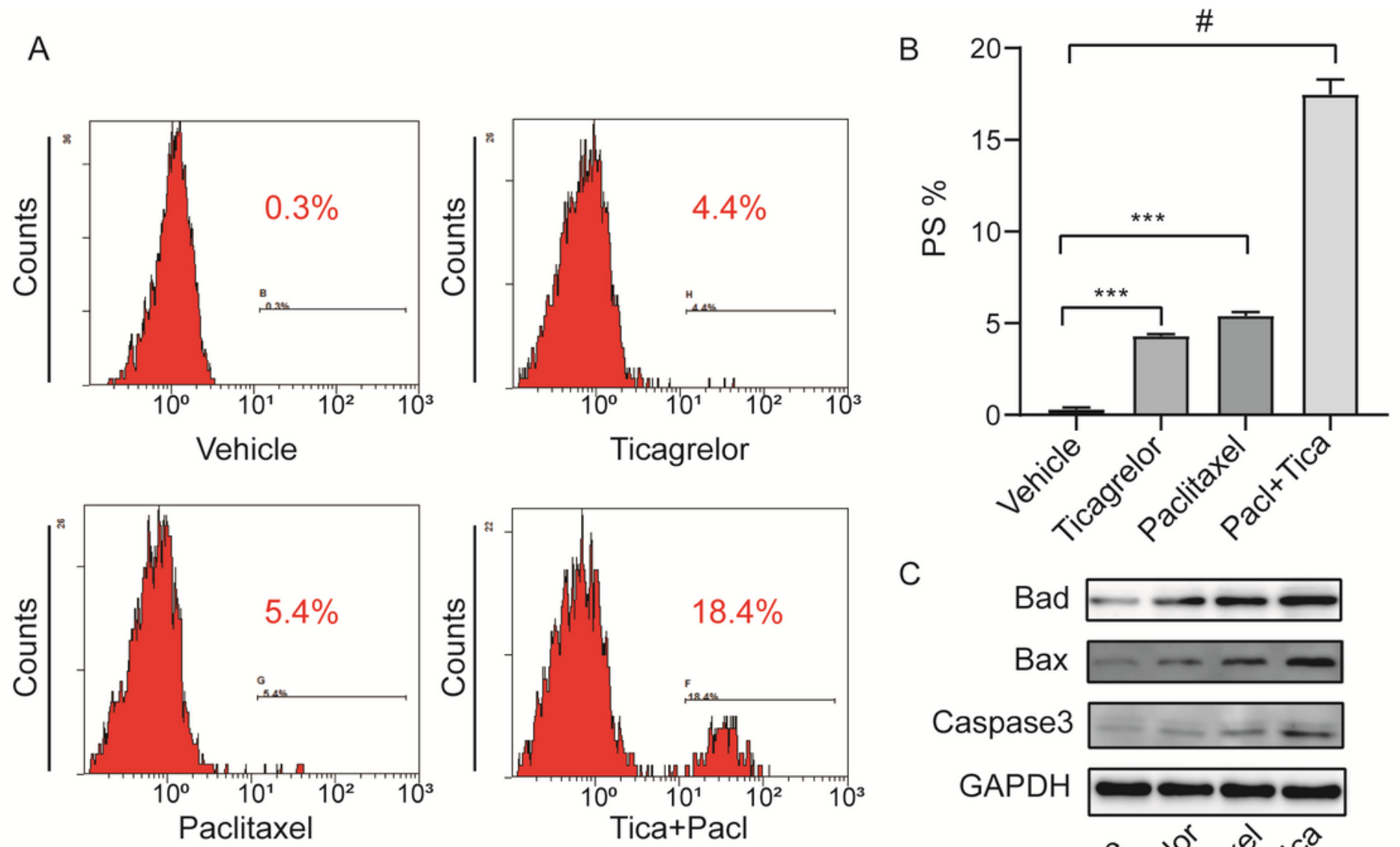

C

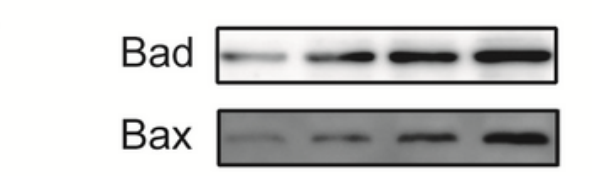

Caspase3

GAPDH

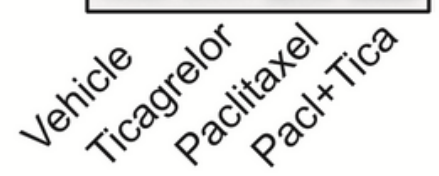

D

E

$\mathrm{F}$
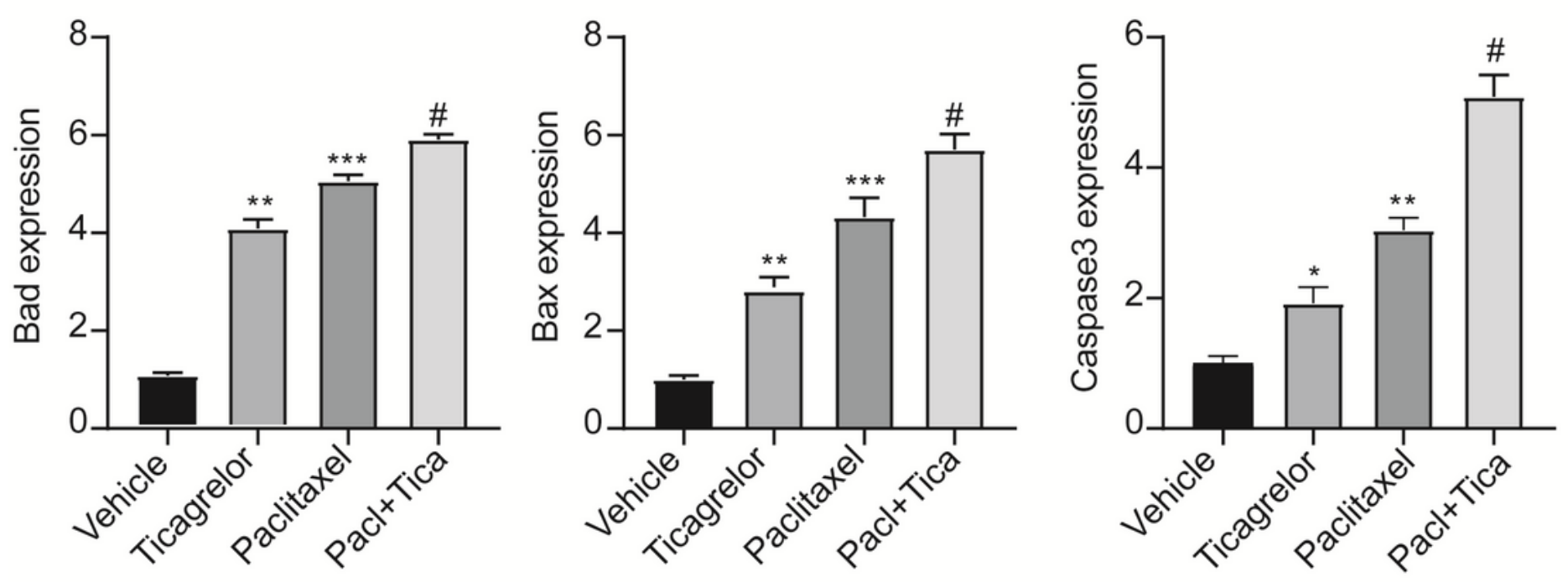

Figure 3 
Combination of paclitaxel with ticagrelor promotes platelet apoptosis. (A, B) Washed mouse platelets were treated with $5 \mathrm{nM}$ paclitaxel, or $20 \mu \mathrm{M}$ ticagrelor, or paclitaxel $(5 \mathrm{nM})+$ ticagrelor $(20 \mu \mathrm{M})$ at room temperature for $1 \mathrm{~h}$. Platelet apoptosis was determined by PS externalization assay. Data are expressed as the mean \pm standard deviation ( $S D ; n=3)$. ${ }^{* *} P<0.001$; $\# P<0.0001$. (C-F) Following PS externalization assay, samples were lysed with RIPA buffer and western blot analysis was carried out using primary antibodies against Bad (dilution, 1:1,000), Bax (dilution, 1:1,000), caspase-3 (dilution, 1:1,000), or GAPDH (dilution, 1:2,000). Images were captured using the Tanon-5200 Multi system. Semi-Quantification was performed using the Image $J$ software. ${ }^{*} P<0.05,{ }^{*} \mathrm{P}<0.01$ and $* \star * P<0.001 ; \# P<0.0001$. PS, phosphatidylserine. 
A

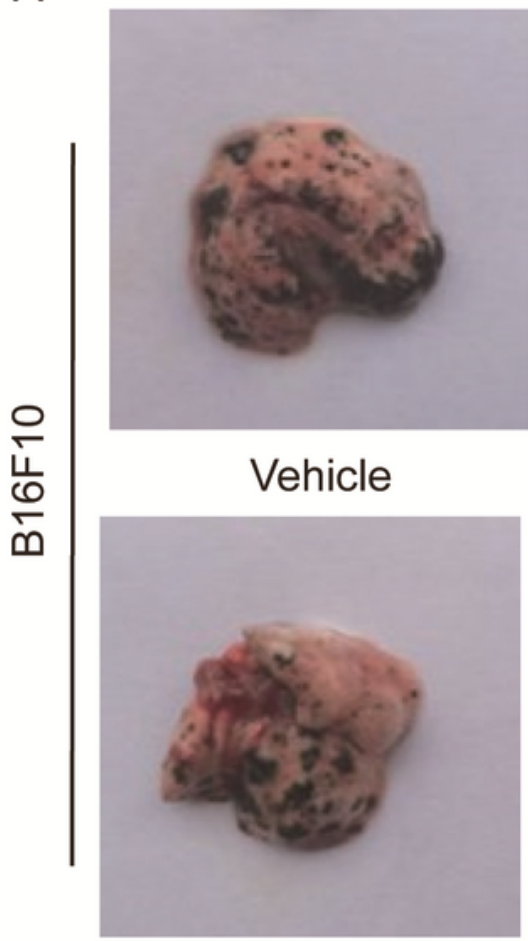

Paclitaxel

C

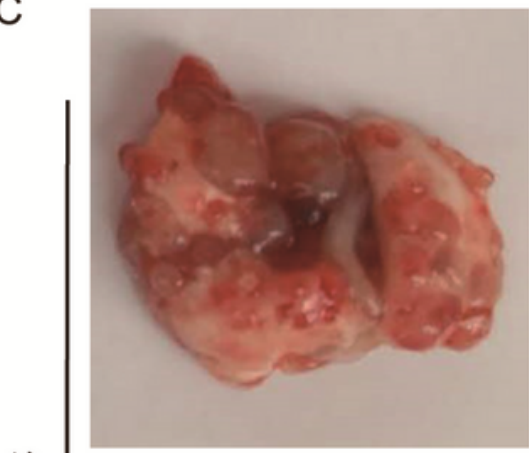

J

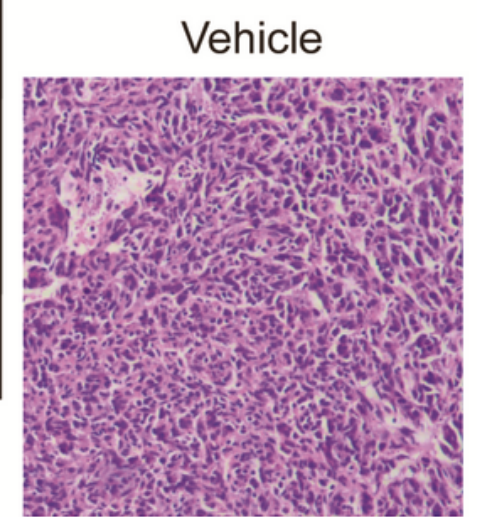

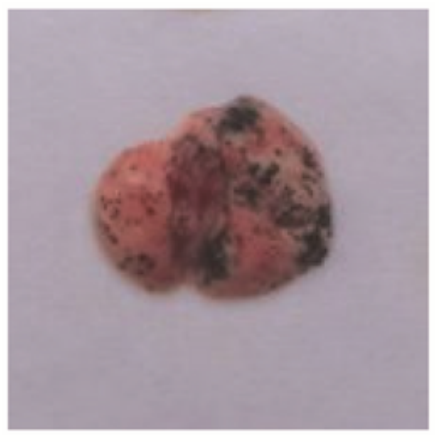

Ticagrelor

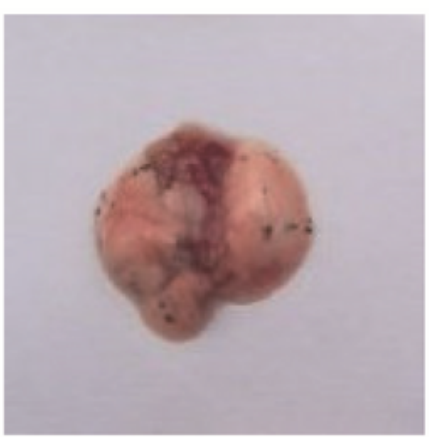

Pacl+Tica

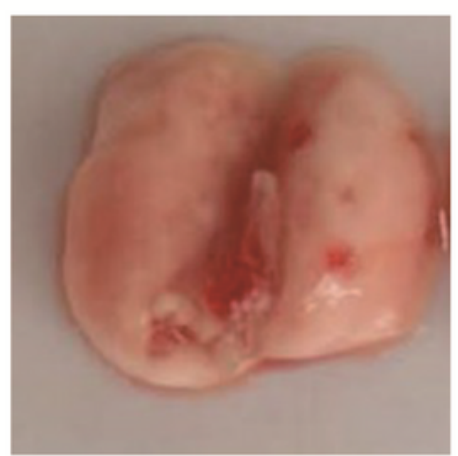

Ticagrelor

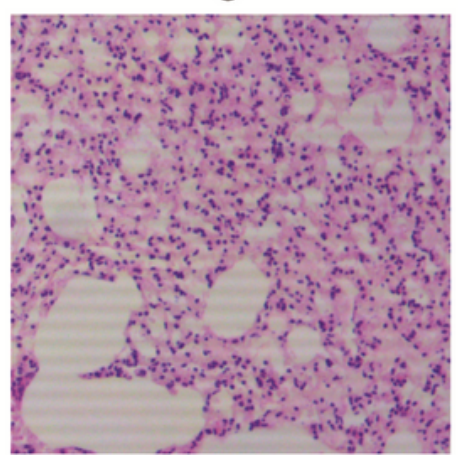

B
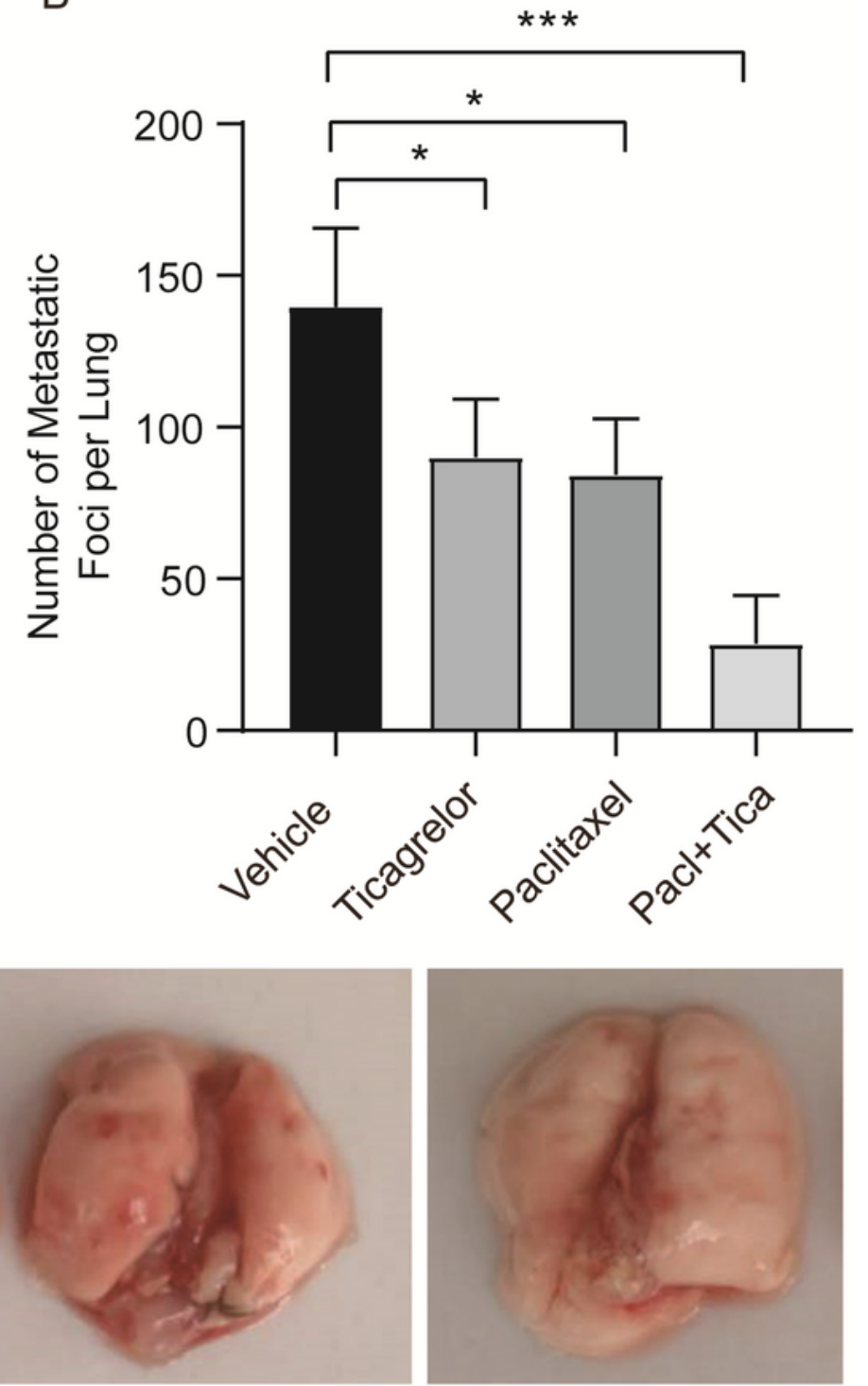

Paclitaxel

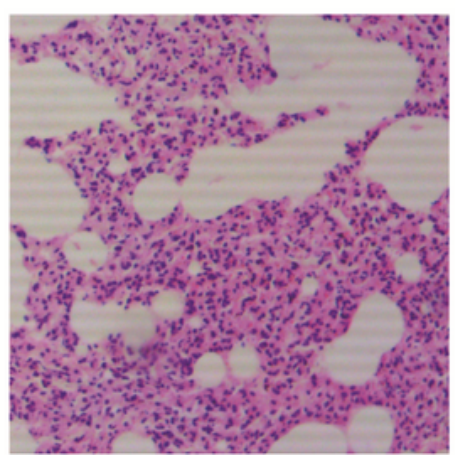

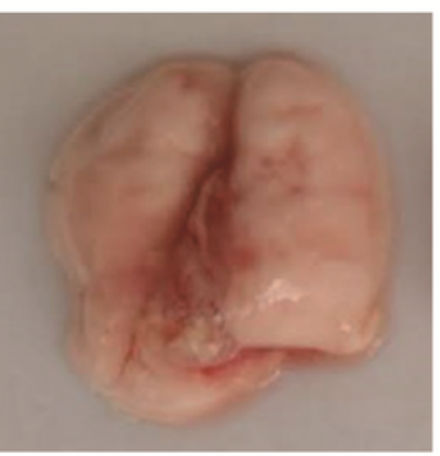

Pacl+Tica

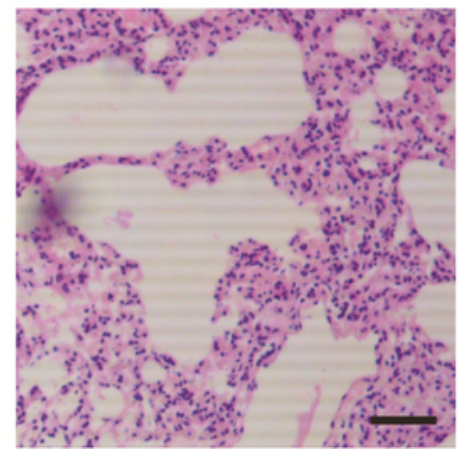

Figure 4

Combination of paclitaxel with ticagrelor inhibits lung metastasis in mice. (A) Two tumor models were established in C57BL/ 6 mice following inoculation of 3x105 B16F10 or 5x105 LLC cells (in $50 \mu \mathrm{l} \mathrm{PBS)}$ into the tail vein (5 mice/group). The next day, mice were administered with paclitaxel $(10 \mathrm{mg} / \mathrm{kg}$ per mouse), ticagrelor (10 mg/kg per mouse), or paclitaxel + ticagrelor by tail vein injection (every 2 days; 5 mice/group). Mice in the control group were injected with PBS. At 15 days after the first injection, the 
lungs were removed. (B) The number of metastatic nodules on the entire lung surface is shown $(n=5)$. ${ }^{*} \mathrm{P}<0.05,{ }^{*} \mathrm{P}<0.01$ and ${ }^{* *} \mathrm{P}<0.001$ vs. the PBS group. (C) At 21 days following treatment of LLC cellinjected mice with paclitaxel (10 mg/kg per mouse), or ticagrelor (10 mg/kg per mouse), or paclitaxel + ticagrelor, the lungs were removed, and H\&E staining was carried out (scale bar, $100 \mu \mathrm{m})$. LLC, Lewis lung carcinoma.

\section{Supplementary Files}

This is a list of supplementary files associated with this preprint. Click to download.

- Supplement1.tif 